\title{
Calmodulin Regulates Transglutaminase 2 Cross-Linking of Huntingtin
}

\author{
Gina M. Zainelli, ${ }^{1}$ Christopher A. Ross, ${ }^{3}$ Juan C. Troncoso,${ }^{4}$ John K. Fitzgerald, ${ }^{2}$ and Nancy A. Muma ${ }^{1}$ \\ Departments of ${ }^{1}$ Pharmacology and ${ }^{2}$ Cell Biology, Neurobiology, and Anatomy, Loyola University Medical Center, Maywood, Illinois 60153, and \\ Departments of ${ }^{3}$ Psychiatry, Neurology, and Neuroscience and ${ }^{4}$ Pathology, The Johns Hopkins University School of Medicine, Baltimore, Maryland 21205
}

Striatal and cortical intranuclear inclusions and cytoplasmic aggregates of mutant huntingtin are prominent neuropathological hallmarks of Huntington's disease (HD). We demonstrated previously that transglutaminase 2 cross-links mutant huntingtin in cells in culture and demonstrated the presence of transglutaminase-catalyzed cross-links in the HD cortex that colocalize with transglutaminase 2 and huntingtin. Because calmodulin regulates transglutaminase activity in erythrocytes, platelets, and the gizzard, we hypothesized that calmodulin increases cross-linking of huntingtin in the HD brain. We found that calmodulin colocalizes at the confocal level with transglutaminase 2 and with huntingtin in HD intranuclear inclusions. Calmodulin coimmunoprecipitates with transglutaminase 2 and huntingtin in cells transfected with myc-tagged N-terminal huntingtin fragments containing 148 polyglutamine repeats (htt-N63-148Qmyc) and transglutaminase 2 but not in cells transfected with myc-tagged N-terminal huntingtin fragments containing 18 polyglutamine repeats. Our previous studies demonstrated that transfection with both htt-N63-148Q-myc and transglutaminase 2 resulted in crosslinking of mutant huntingtin protein fragments and the formation of insoluble high-molecular-weight aggregates of huntingtin protein fragments. Transfection with transglutaminase 2 and htt-N63-148Q-myc followed by treatment of cells with $N$-(6-aminohexyl)-1naphthalenesulfonamide, a calmodulin inhibitor, resulted in a decrease in cross-linked huntingtin. Inhibiting the interaction of calmodulin with transglutaminase and huntingtin protein could decrease cross-linking and diminish huntingtin aggregate formation in the HD brain.

Key words: calmodulin; Huntington; transglutaminase; protein aggregation; inclusion bodies; cross-linking

\section{Introduction}

Huntington's disease (HD) is an autosomal dominant neurodegenerative disorder caused by an unstable CAG triplet repeat expansion in the open reading frame of the first exon encoding a polyglutamine stretch in the $\mathrm{N}$ terminus of the huntingtin protein. Neuropathological hallmarks of HD include perinuclear cytoplasmic aggregates and intranuclear inclusions of mutant huntingtin (Davies et al., 1997; DiFiglia et al., 1997; Becher et al., 1998; Gourfinkel-An et al., 1998; Ross et al., 1998; Gutekunst et al., 1999). A current hypothesis is that the calcium-dependent enzyme transglutaminase cross-links huntingtin protein to form aggregates and intranuclear inclusions.

Transglutaminases are a family of calcium-activated enzymes that catalyze the covalent cross-linking of peptide-bound glutamine residues of substrate proteins to the $\epsilon$-amino-group of lysine residues on substrate proteins (Hand et al., 1993; Lorand, 1996; Cooper et al., 1999). The resulting isodipeptide bonds can occur between or within substrate proteins, leading to conforma-

\footnotetext{
Received Sept. 30, 2003; revised Dec. 16, 2003; accepted Dec. 17, 2003.

This work was supported by a grant from the Huntington's Disease (HD) Society of America, and human tissues were provided through National Institutes of Health Grants NS16375 (HD Center, C.A.R., program director) and MH/NS31862 (Harvard Brain Tissue Resource Center).

Correspondence should be addressed to Dr. Nancy A. Muma, Loyola University Medical Center, Department of Pharmacology, 2160 South First Avenue, Maywood, IL 60153. E-mail: nmuma@lumc.edu.

D01:10.1523/JNEUROSCI.4424-03.2004

Copyright $\odot 2004$ Society for Neuroscience $\quad 0270-6474 / 04 / 241954-08 \$ 15.00 / 0$
}

tional changes, dimers, or multimeric complexes (Selkoe et al., 1982; Hand et al., 1993; Lorand, 1996).

Huntingtin with $>40$ CAG repeats is an excellent substrate for transglutaminase 2 in vitro and in cell culture (Cariello et al., 1996; Gentile et al., 1998; Kahlem et al., 1998; Cooper et al., 1999; Karpuj et al., 1999; Violante et al., 2001). An increase in transglutaminase enzymatic activity and transglutaminase 2 protein has been demonstrated in HD cases compared with controls (Karpuj et al., 1999; Lesort et al., 1999). Huntingtin and transglutaminase 2 and huntingtin and transglutaminase-catalyzed cross-links colocalize in HD brain tissue, and transglutaminase 2 cross-links huntingtin in cells in culture (Zainelli et al., 2003).

Calmodulin regulates transglutaminase in systems such as the human erythrocyte cytoskeleton (Billett and Puszkin, 1991), human platelets, and the chicken gizzard (Plank et al., 1983). In these systems, calmodulin increased transglutaminase activity. Calmodulin is a $17 \mathrm{kDa}$ protein that activates a host of enzymes during calcium binding (Cheung, 1982). A membrane-associated erythrocyte transglutaminase cross-links substrates at physiological and lower than physiological calcium concentrations in the presence of calmodulin (Billett and Puszkin, 1991). Total transglutaminase activity is threefold higher in the presence of calmodulin in human platelets and the chicken gizzard (Puszkin and Raghuraman, 1985). It has also been demonstrated that huntingtin binds strongly to calmodulin-Sepharose in the presence of calcium, indicating that it may interact with huntingtin in vivo 
Table 1. HD and control brain tissue used in immunofluorescence experiments

\begin{tabular}{clll}
\hline & Age (years) & Gender & Postmortem interval (hours) \\
\hline HD cases & & & \\
1 & 71 & Male & 20.5 \\
2 & 46 & Female & 18.8 \\
3 & 67 & Female & 23.6 \\
4 & 62 & Female & 21.0 \\
Control cases & & & \\
1 & 53 & Male & 20.0 \\
2 & 62 & Male & 12.0 \\
3 & 67 & Male & 20.0 \\
4 & 73 & Male & 24.0 \\
\hline
\end{tabular}

as well (Bao et al., 1996). Mutant huntingtin protein may bind to calmodulin and then activate transglutaminase by bringing the proteins into close enough proximity to interact. Thus, calmodulin may also act to regulate transglutaminase cross-linking activity in HD.

We examined the colocalization of both huntingtin protein and the transglutaminase-catalyzed isopeptide bonds with calmodulin in intranuclear inclusions in HD. Lastly, we examined the association of transglutaminase 2 and huntingtin with calmodulin and the effects of a calmodulin inhibitor on transglutaminase-induced cross-linking of huntingtin in cells in culture.

\section{Materials and Methods}

Human brain tissue. Control brain tissue (free from neurologic disease) was obtained from the Loyola University Brain Bank (Table 1). HD brain tissue was obtained from the Harvard Brain Tissue Resource Center and the Brain Bank at The Johns Hopkins University School of Medicine. The institutional protocols for sampling, processing, and storing specimens are similar enough to quantitatively compare samples from these institutions. Tissue is blocked, frozen in isopentane, cooled on dry ice or on glass plates, and then stored at $-80^{\circ} \mathrm{C}$. Frozen postmortem human cortical (Brodman's areas 45, 46, or area 9) brain tissue was obtained from Vonsattel's (Vonsattel et al., 1983) grade-three HD subjects for immunofluorescent labeling and immunoblot experiments. The following samples were used for immunofluorescent labeling experiments: HD subjects $[n=4$; mean age, $61.5 \pm 5.48$ (SEM); mean postmortem interval (PMI), $20.98 \pm 1.99 \mathrm{hr}(\mathrm{SEM})]$ and age-matched control subjects $[n=4$; mean age, $63.75 \pm 4.23(\mathrm{SEM})$; PMI, $19.00 \pm 2.52 \mathrm{hr}(\mathrm{SEM})]$. A $t$ test showed no significant difference between the mean age $(p=0.76)$ and PMI ( $p=0.59$ ) of control versus HD tissue used in the immunofluorescent labeling experiments.

Antibodies. The monoclonal antibody (mAb) 81D4 (mouse IgM) directed against the transglutaminase-induced cross-links was used at a 1:300 dilution for immunofluorescence experiments (CovalAb, Lyon, France). mAb2166 (mouse IgG) is a monoclonal antibody to the $\mathrm{N}$ terminus of the huntingtin protein sequence from amino acid 181-812 (outside of the polyglutamine domain) that was used at a dilution of 1:100 for immunofluorescence (Chemicon, Temecula, CA). This antibody was reported to recognize both the normal and mutant forms of huntingtin on Western blots (Trottier et al., 1995; Kahlem et al., 1998) and to recognize huntingtin in the perikarya of neurons as punctate staining (Trottier et al., 1995). The antibody to transglutaminase 2, TG100 (mouse IgG), was used at a dilution of 1:100 for immunofluorescence and immunoblots (NeoMarkers, Union City, CA). The monoclonal antibody to calmodulin, Cam I, was used at a dilution of 1:5000 on immunoblots (Santa Cruz Biotechnology, Santa Cruz, CA). The monoclonal antibody against calmodulin, Cam85 (Zymed, South San Francisco, CA), was used for immunofluorescence experiments at a dilution of 1:100. The $\mathrm{mAb}$ 81D1c2 (mouse $\mathrm{IgG}$ ) directed against the transglutaminaseinduced cross-links was used at a 1:500 dilution for immunoblots (Co$\mathrm{val} A \mathrm{~b})$. The anti-myc antibody conjugated to horseradish peroxidase (HRP) was used at a dilution of 1:2500 on immunoblots (Invitrogen,
Carlsbad, CA). An antibody to actin was used at a dilution of 1:1000 for immunoblots (Sigma, St. Louis, MO).

Double-label immunofluorescence microscopy. Sections of fresh frozen postmortem human brain tissue ( $16 \mu \mathrm{m}$ thick) from each Huntington's disease case and control cases were mounted on glass slides. The sections were then fixed in $70 \%$ ethanol- $0.9 \% \mathrm{NaCl}$. After blocking nonspecific binding with 5\% normal goat serum (NGS), sections were incubated overnight in antibody directed to calmodulin, Cam85. Next, sections were washed and incubated in goat anti-mouse (IgG fragment crystalizable constant region chain specific, 1:100) conjugated to fluorescein isothiocyanate (FITC) (Jackson ImmunoResearch, West Grove, PA). Tissue sections were again incubated in 5\% NGS to block nonspecific binding, incubated overnight in the second primary antibody $81 \mathrm{D} 4$, and then incubated in goat anti-mouse IgM antibody ( $\mu$-chain specific, which recognizes 81D4 IgM, 1:100) conjugated to Rhodamine Red-X.

Another group of tissue sections was labeled with the primary antibody, Cam85, incubated with rabbit anti-mouse fab fragment to convert recognition of the primary antibodies from IgG to a rabbit species, and then incubated with goat anti-rabbit $(1: 100)$ conjugated to FITC (Jackson ImmunoResearch). This was done to prevent labeling of the two IgG subclass primary antibodies (mAb2166 and TG-100) with the same secondary antibody. Sections were then incubated overnight in the second primary antibody, mAb2166 (directed against the $\mathrm{N}$ terminus of the huntingtin protein), or TG-100 (directed against transglutaminase 2). Sections were washed and incubated in goat anti-mouse conjugated to Rhodamine Red-X at a dilution of 1:00 (Jackson ImmunoResearch).

The tissue sections were incubated in 1\% Sudan Black B for 10 min to reduce the autofluorescence caused by lipofuscin (Schnell et al., 1999). Next, coverslips were mounted on tissue sections with media containing 4',6'-diamidino-2-phenylindole (DAPI), a dye that specifically labels DNA and allows for the visualization of nuclei. As a control for nonspecific labeling with secondary antibody, omission of primary antibodies was also performed on a slide from each case (see Fig. $1 A, B$ ). A preadsorption control was also performed for the $81 \mathrm{D} 4$ antibody to determine its specificity. The specificity of the cross-link antibody, 81D4, was also demonstrated in previous studies (El Alaoui et al., 1992; Norlund et al., 1999). For example, in vitro incubation of tau protein with transglutaminase produced cross-linked tau that could be immunoprecipitated using 81D4 (Norlund et al., 1999).

Fluorescence and confocal microscopy. Tissue sections were examined using a U-MCB fluorescence microscope (Olympus Optical, Tokyo, Japan) as well as an LSM 510 Axiovert laser scanning confocal microscope (Zeiss, Thornwood, NY) to determine colocalization of antibody labeling. We examined the following combinations of antibodies: (1) crosslink epitopes (labeled with Rhodamine Red-X) and calmodulin epitopes (labeled with FITC), (2) transglutaminase 2 epitopes (labeled with Rhodamine Red-X) and calmodulin epitopes (labeled with FITC), and (3) cross-link epitopes (labeled with Rhodamine Red-X) and calmodulin epitopes (labeled with FITC). Nuclei were visualized using DAPI under ultraviolet illumination.

The Axiovert laser scanning confocal microscope (Zeiss) has a twotrack system in which the lasers are never on at the same time, thus eliminating interference of one filter set with another and eliminating bleed-through fluorescence. For each capture, the microscope imaging system generated a three-channel (RGB), $512 \times 512$ pixel composite image. The three channels of each image correspond to FITC or Rhodamine Red-X and an overlaid colocalized image. Specifically, nuclei that contained fluorescent labeling were analyzed for colocalization at the confocal level within a $0.8-\mu \mathrm{m}$-thick slice. Four control and four HD cases were examined, and confocal images were obtained. One hundred calmodulin-positive nuclei ( 25 nuclei from each of four HD cases) were examined in each set of slides to determine the number that also contained the huntingtin epitope, the transglutaminase 2 epitope, and the cross-link epitope.

Cell culture system. Human embryonic kidney 293T (HEK 293T) cells were grown in the presence of $5 \% \mathrm{CO}_{2}$ and in OptiMEM reduced-serum medium with HEPES buffer, L-glutamine, and sodium bicarbonate (Invitrogen). Nonessential amino acids, antimycotic and antibiotic agents, and $10 \%$ FBS were also added to the medium. Cells were grown to $40-$ 
$50 \%$ confluence on $100 \mathrm{~mm}^{2}$ culture dishes and transfected using Lipofectamine Plus (Invitrogen). Transfection of the following constructs was performed in various combinations: pcDNA3.1 as a vector control, myctagged $\mathrm{N}$-terminal huntingtin fragments containing 18 polyglutamine repeats (htt-N63-18Q-myc) and myc-tagged N-terminal huntingtin fragments containing 148 polyglutamine repeats (htt-N63-148Q) in pcDNA3.1, and transglutaminase 2 in pcDNA3.1. Twenty-four hours after transfection, cells were treated with various concentrations of $N$-(6aminohexyl)-1-naphthalenesulfonamide (w5-hydrochloride; Tocris Cookson, Ballwin, MO) in some experiments. Forty-eight hours after transfection, cells were harvested and resuspended in aggregate lysis buffer containing $50 \mathrm{~mm}$ Tris- $\mathrm{HCl}, \mathrm{pH} 8.8,100 \mathrm{~mm} \mathrm{NaCl}, 5 \mathrm{~mm} \mathrm{MgCl}_{2}$, $0.05 \%$ NP-40, and 1 mm EDTA (Hazeki et al., 2000). The cell lysates were then either run directly on Western blots, or a low-solubility fraction was prepared. The low-solubility fraction was prepared by centrifugation of the lysate at $12,000 \times g$ for $5 \mathrm{~min}$ and removal of the supernatant. The pellet was then resuspended in 95\% formic acid and incubated for $40 \mathrm{~min}$ at $37^{\circ} \mathrm{C}$ in a shaking water bath, and then the formic acid was removed under a vacuum (Hazeki et al., 2000). The pellets were then resuspended in sample loading buffer and run on gels or resuspended in immunoaffinity purification buffer for additional analysis.

Immunoaffinity purification. Immunoaffinity purification of proteins containing transglutaminase-induced $\epsilon$ - $(\gamma$-glutamyl) lysine cross-links from $200 \mu \mathrm{g}$ of low-solubility protein lysate $(1 \mu \mathrm{g} / \mu \mathrm{l})$ was performed using Sepharose beads prebound to the 81D4 antibody using a protocol developed by CovalAb and as described previously (Norlund et al., 1999).

Immunoprecipitation with calmodulin-Sepharose. The low-solubility fractions from lysates from the transfection of htt-N63-148Q-myc, httN63-18Q-myc, transglutaminase 2, and vector combinations were immunoprecipitated with immobilized bovine brain calmodulin (Calbiochem, La Jolla, CA). A modification of the procedure was followed. First, the immobilized calmodulin was washed with buffer containing (in $\mathrm{M}$ ) 0.1 calcium phosphate, 0.1 magnesium chloride, and 0.1 Tris- $\mathrm{HCl}, \mathrm{pH}$ 7.5. Samples were incubated with immobilized calmodulin for $1.5 \mathrm{hr}$ with gentle shaking. Next, immobilized calmodulin was washed for $5 \mathrm{~min}$ and centrifuged for $5 \mathrm{~min}$ for a total of four washes. Elution of proteins that bound to calmodulin was performed by addition of elution buffer containing (in M) 0.1 EGTA and 0.1 Tris-HCl, pH 7.4.

Immunoblots. Cell lysates and immunoaffinity-purified protein lysates were analyzed using 3-8\% Tris-acetate denaturing gels for cell-culture experiments (Novex system; Invitrogen) and then electrophoretically transferred to nitrocellulose membranes. Nonspecific antibody labeling was blocked with a solution of 5\% nonfat dry milk in Tris-buffered saline with $0.05 \%$ Triton X-100. After overnight incubation in primary antibody, blots incubated with TG-100, calmodulin, or actin antibodies were either incubated in goat anti-mouse secondary antibody conjugated to horseradish peroxidase (Cappel, Aurora, $\mathrm{OH}$ ) or incubated with peroxidase-antiperoxidase (Sigma). Autoradiograms were produced using the ECL advanced chemiluminescence reagents. Blots incubated with the $\alpha$-myc antibody conjugated to HRP were washed and then directly incubated with ECL reagents (Amersham Biosciences, Buckinghamshire, UK). Experiments were performed in triplicate with similar results.

Treatment with w5-hydrochloride. To determine whether calmodulin regulates transglutaminase cross-linking activity and aggregate formation, we used the calmodulin inhibitor w5-hydrochloride (Tocris Cookson) at concentrations ranging from 50 to $1000 \mu \mathrm{M}$ in the cell culture system. We determined whether the calmodulin antagonist affected aggregate formation in cells by immunoaffinity purification of crosslinked, insoluble proteins and Western blotting. We then used antibodies against huntingtin (anti-myc antibody) to recognize cross-linked huntingtin proteins on immunoblots. Transglutaminase and huntingtin expression were verified using the transglutaminase antibody and $\alpha$-myc antibody (recognizes huntingtin) on immunoblots.

Digital fluorescence imaging of intracellular calcium levels. HEK 293T cells were grown on $25 \mathrm{~mm}$ glass coverslips that were coated with collagen. Cells were loaded with $1 \mu \mathrm{M}$ of the calcium-sensitive indicator fura-2 AM (Molecular Probes, Eugene, OR) (Takahashi et al., 1999) in HBSS for $15 \mathrm{~min}$ at $37^{\circ} \mathrm{C}$. The coverslips were rinsed twice with HBSS and then fit into a holder. The holder was filled to $1 \mathrm{ml}$ with warm HBSS and mounted onto the stage of the Axiovert 135 inverted microscope (Zeiss). At least 15 regions of interest (containing multiple cells each) were monitored for calcium measurements using a $40 \times$ oil objective on the microscope. Digital images were visualized on a computer monitor and captured using a two-camera system. The excitation emission from a xenon lamp alternates between $340 \mathrm{~nm}$ (calcium bound) and $380 \mathrm{~nm}$ (calcium free), and emission was measured at $520 \mathrm{~nm}$. After at least $180 \mathrm{sec}$ of baseline calcium fluorescence intensity, w5-hydrochloride was added in successive aliquots of molar concentrations of 50 and $100 \mu \mathrm{m}$ without washing out drug added previously. These additions resulted in a $>1 \mathrm{~mm}$ final concentration of w5-hydrochloride added with no alteration of intracellular calcium levels. As a positive control, ionomycin was added at a concentration of $2.5 \mu \mathrm{M}$. Fluorescent signals were measured for a change in the fura- 2 ratio. Fluorescent signals were obtained, saved, and analyzed using the Attofluor RatioVision System (Zeiss).

\section{Results}

\section{Calmodulin colocalizes with proteins in intranuclear inclusions in the HD brain}

Twenty-five nuclei from each of four different HD cases were examined for the colocalization of calmodulin and either transglutaminase 2, huntingtin, or the transglutaminase-induced $\epsilon$-( $\gamma$-glutamyl $)$ lysine cross-link. There was a very high degree of colocalization of calmodulin protein with transglutaminase 2, cross-links, and huntingtin proteins. A total of $98 \%$ of huntingtin-positive nuclei colabel with the calmodulin antibody, whereas $100 \%$ of calmodulin-positive nuclei also label with the huntingtin antibody (Fig. 1D). A total of $99 \%$ of calmodulinpositive nuclei colocalize with transglutaminase 2 epitopes, whereas $100 \%$ of transglutaminase 2-positive nuclei colocalize with calmodulin epitopes (Fig. $1 E$ ). Finally, 100\% of cross-linkpositive nuclei colocalize with calmodulin epitopes, whereas $100 \%$ of calmodulin-positive nuclei colocalize with cross-link epitopes (Fig. 1C).

\section{Immunoprecipitation of calmodulin and transglutaminase 2} HEK 293T cells were transfected with the following: (1) huntingtin containing a normal-length glutamine domain (i.e., htt-N6318Q-myc) and vector, (2) transglutaminase 2 and htt-N63-18Qmyc, (3) huntingtin with a polyglutamine domain expansion (i.e., htt-N63-148Q-myc) and vector, or (4) transglutaminase 2 and htt-N63-148Q-myc. The insoluble fractions from these lysates were immunoprecipitated using anti-calmodulin-Sepharose and run directly on immunoblots. Calmodulin was only found in the insoluble fraction from cells transfected with transglutaminase 2 and htt-N63-148Q-myc; calmodulin was not in the insoluble fraction from cells transfected with transglutaminase 2 and htt-N6318Q-myc, cells transfected with htt-N63-148Q-myc and vector, or cells transfected with htt-N63-18Q-myc and vector (Fig. 2A). Transglutaminase 2 coimmunoprecipitates with calmodulin from cells cotransfected with transglutaminase 2 and htt-N63-148Q-myc (Fig. $2 B$ ). Transglutaminase 2 did not coimmunoprecipitate from cells cotransfected with htt-N63-18Q-myc and vector, cells transfected with htt-N63-18Q-myc and transglutaminase 2, or cells cotransfected with htt-N63-148Q-myc and vector (Fig. 2 B). Two bands are present in the immunoblots prepared with the huntingtin antibody (Fig. 2C). One band is in the lane corresponding to cotransfection of vector and htt-N63-148Q-myc, and the other band is in the lane corresponding to cotransfection of transglutaminase 2 and htt-N63148Q-myc (Fig. 2C). Calmodulin protein was loaded directly on the gel for use as a positive control for the immunoblots (Fig. 2A).

\section{Treatment with w5-hydrochloride}

Twenty-four hours after transient cotransfection of transglutaminase 2 and htt-N63-148Q-myc, cells were treated with 0,50 , 
$\mathbf{A}$

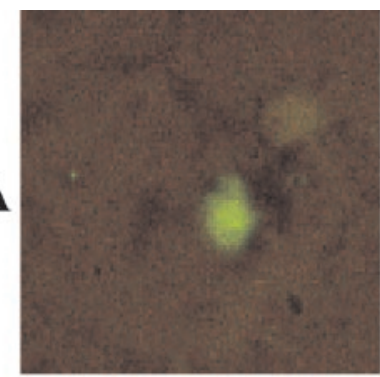

calmodulin
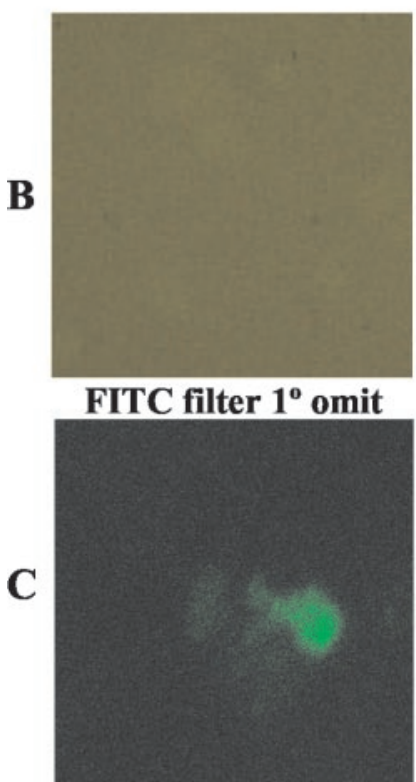

calmodulin

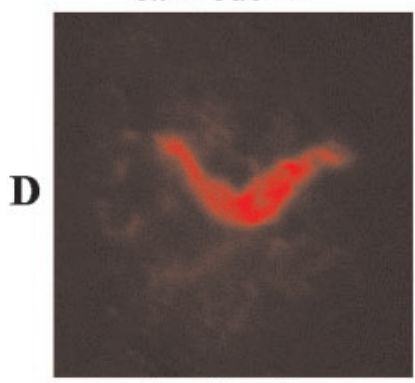

huntingtin

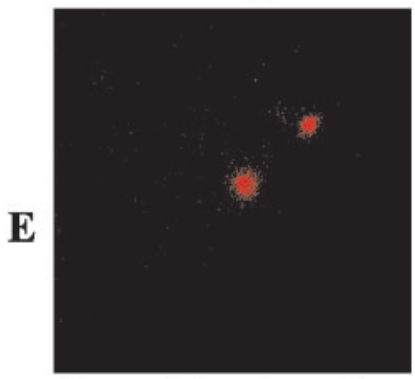

transglutaminase 2

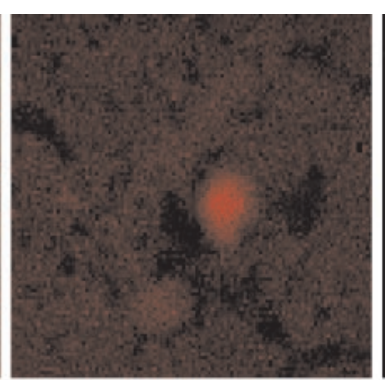

huntingtin

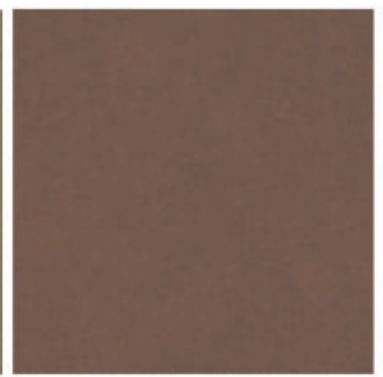

RhRed filter $1^{\circ}$ omit

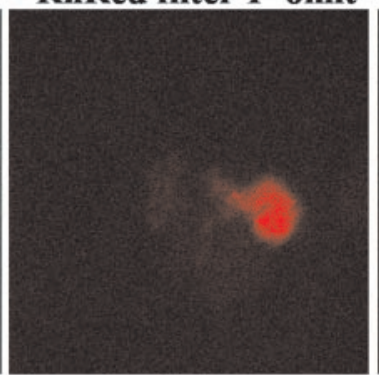

cross-link

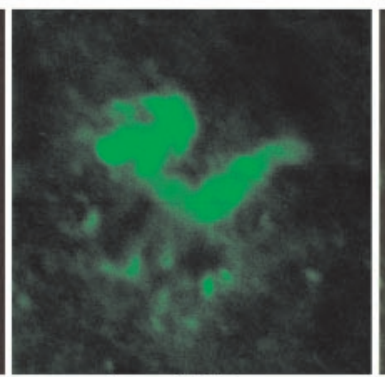

calmodulin

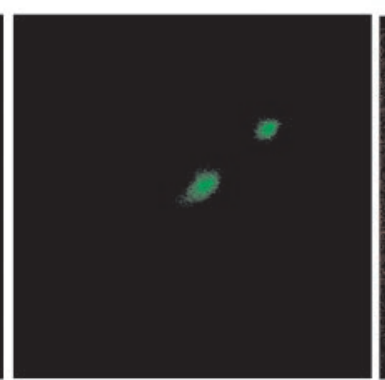

calmodulin

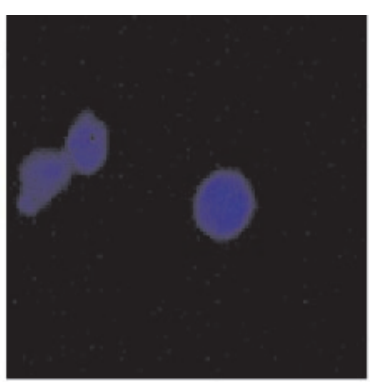

nucleus

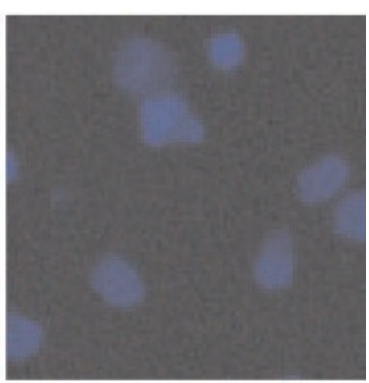

nucleus

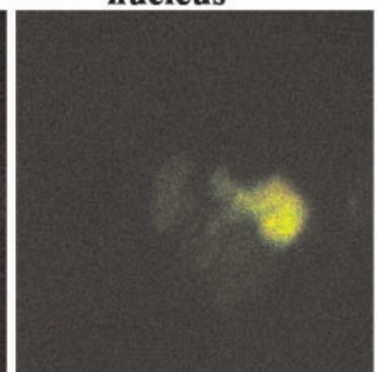

co-localization

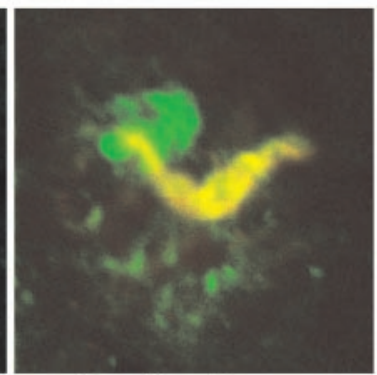

\section{co-localization}

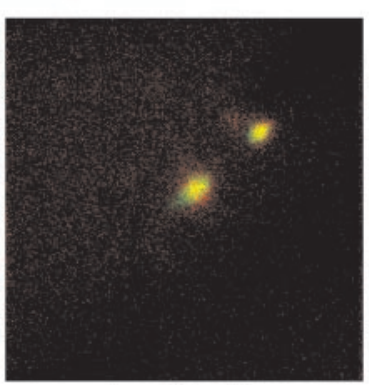

co-localization
Figure 1. Nuclei that contained intranuclear inclusions were first localized by fluorescence microscopy using DAPI. Colocalization of each epitope was examined by fluorescence microscopy, and control slides were produced in which both primary antibodies were omitted (each separately as well as both combined) from the assay to ensure that the secondary antibodies did not cross-react in a nonspecific manner. For example, colocalization of calmodulin and huntingtin was examined in $A$. In the same experiment, FITC fluorescent labeling was examined after omission of the calmodulin primary $\left(1^{\circ}\right)$ antibody and Rhodamine Red-X (RhRed) fluorescent labeling was examined after omission of the huntingtin primary antibody $(B)$. Confocal images of intranuclear inclusions ( $C-E$ ) depict labeling of each antibody and colocalization of epitopes (yellow color). $A, B, 1000 \times$ final magnification; $C-E, 4000 \times$ magnification.
100 , or $500 \mu \mathrm{M}$ w5-hydrochloride, a calmodulin inhibitor. Transfection of both transglutaminase 2 and htt-N63-148Qmyc resulted in cross-linking of the huntingtin protein, as did htt-N63-148Q-myc alone (Fig. $3 A$, first and second lanes, respectively). As a result of treatment with the calmodulin inhibitor, there were not observable levels of cross-linked huntingtin proteins detectable on the immunoblots with any of the doses of w5hydrochloride administered (Fig. 3A).

To determine whether w5-hydrochloride regulates transglutaminase cross-linking directly and not by altering intracellular calcium levels, we measured intracellular levels of calcium in untransfected cells using the fluorescent ratiometric calcium indicator fura-2 AM. (Molecular Probes) (Takahashi et al., 1999). We found that doses of w5hydrochloride (50 and $100 \mu \mathrm{M}$ ) that prevent cross-linking of huntingtin did not significantly alter the intracellular calcium levels (Fig. 3D). Doses up to $1000 \mu \mathrm{M}$ did not alter intracellular calcium levels (data not shown).

\section{Discussion}

The goal of this study was to determine whether calmodulin is involved in the pathogenesis of HD. We found that calmodulin is present in intranuclear inclusions in HD cortical tissue. We also observed a high degree of colocalization of calmodulin with huntingtin, transglutaminase 2 , and the transglutaminaseinduced cross-links within nuclei in the HD cortex. These findings suggest that calmodulin associates with and may be a component of intranuclear inclusions. Previous studies demonstrated that transglutaminase selectively cross-links pathological-length huntingtin $(>40 \mathrm{Q})$ over huntingtin with normal-length glutamine domains (Cariello et al., 1996; Gentile et al., 1998; Kahlem et al., 1998; Karpuj et al., 1999). It was also demonstrated previously that huntingtin proteins that have expanded glutamine domains bind to calmodulinSepharose with greater affinity than huntingtin with normal-length glutamine domains (Bao et al., 1996).

Our studies demonstrate that transglutaminase and calmodulin and polyglutamine-expanded huntingtin and calmodulin coimmunoprecipitate from the insoluble fraction of cells that were cotransfected with htt-N63-148Q-myc and transglutaminase 2 but do not coimmunoprecipitate in cells that were transfected with huntingtin containing a normal-length glutamine domain (httN63-18Q-myc). The anti-calmodulin antibody labeled the calmodulin protein in the 
IP: $\alpha$-calmodulin

A IB: $\alpha$-calmodulin

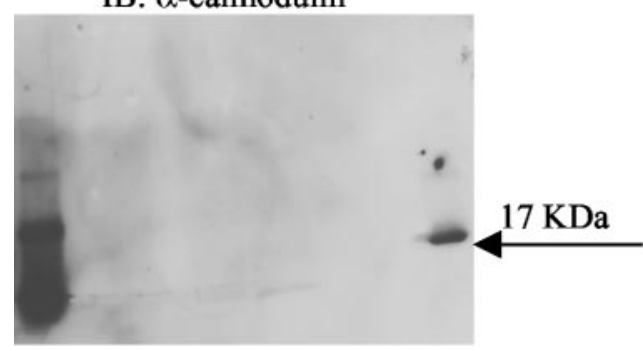

IP: $\alpha$-calmodulin

B IB: TG-100 (TGase 2)

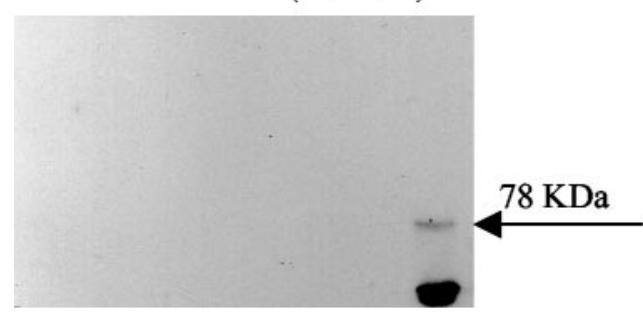

IP: $\alpha$-calmodulin

C IB: $\alpha$-myc (huntingtin)
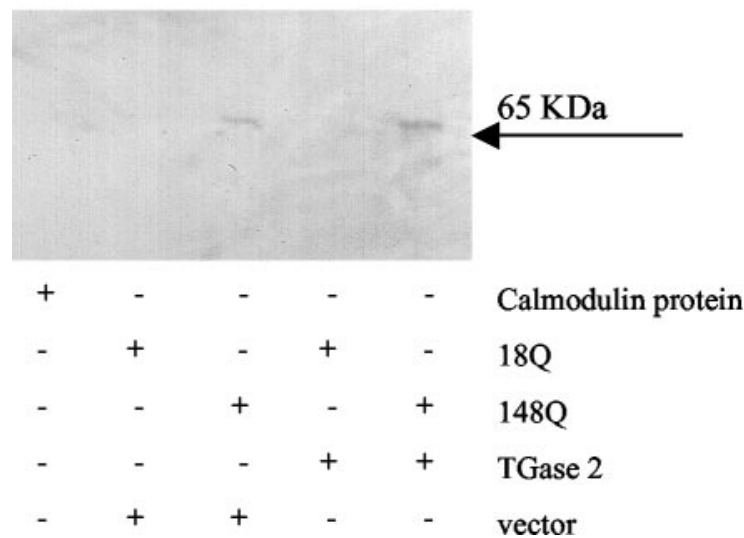

Figure 2. Insoluble fractions of proteins from lysates of cotransfections were solubilized with formic acid. Samples were then immunoprecipitated (IP) with the anti-calmodulin antibody and examined on immunoblots (IBs). Antibodies to calmodulin $(A)$, transglutaminase 2 $(B)$, and huntingtin ( $\alpha$-myc) ( $C$ ) were used on the immunoblots. Calmodulin protein was also directly loaded in the first lane of the gels as a positive control. TGase 2, Transglutaminase 2.

control lane and calmodulin in the insoluble fraction from cotransfection of polyglutamine-expanded huntingtin and transglutaminase 2 but not from cotransfection of polyglutamine-expanded huntingtin and vector (Fig. 2A). The myc-positive band representing cotransfection of polyglutamine-expanded huntingtin and transglutaminase 2 was consistently darker than the band representing cotransfection of polyglutamine-expanded huntingtin and vector (Fig. $2 C$ ). The low levels of endogenous transglutaminase 2 could play a role in the weaker association of calmodulin and huntingtin in the cells not transfected with transglutaminase 2 . The observation of a lighter band in the lane representing cotransfection of polyglutamine-expanded huntingtin and vector compared with huntingtin and transglutaminase 2 (Fig. 2C) suggests that there is less huntingtin associated with calmodulin in the cells and could also explain why there was no visible calmodulin immunoreactivity from the cotransfection of polyglutamine-expanded huntingtin and vector com-
$\mathbf{A}$
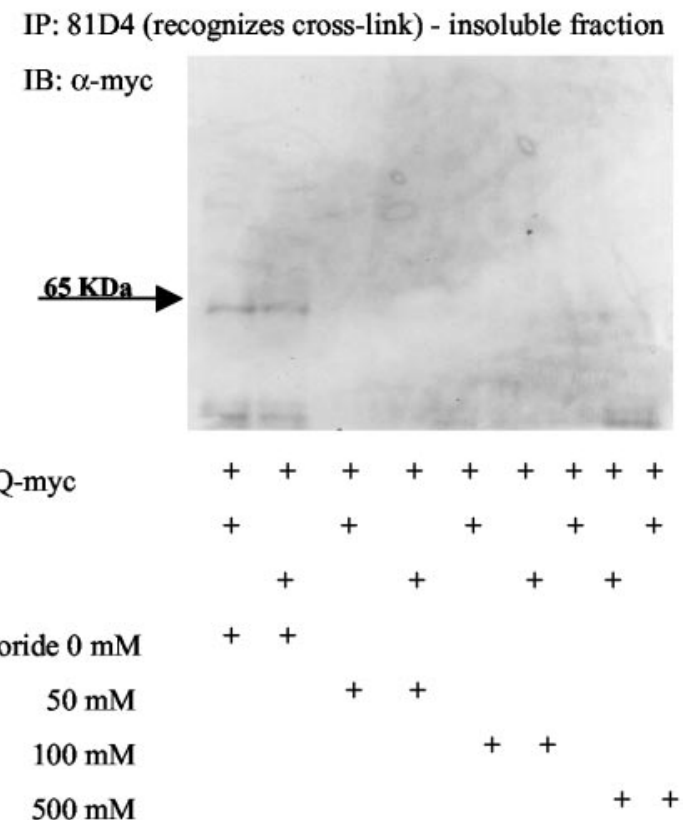

B

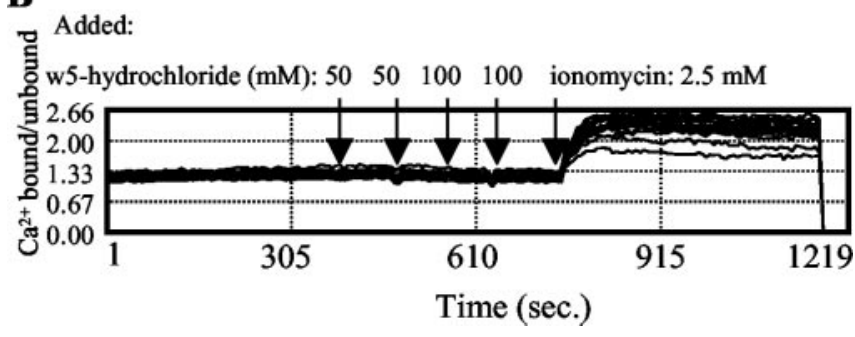

Figure 3. Cells transiently transfected with htt-N63-1480-myc and transglutaminase 2 (TGase 2) or htt-N63-1480-myc and vector were treated with increasing concentrations of the calmodulin inhibitor w5-hydrochloride $(A)$. The insoluble fraction of lysates was solubilized with formic acid, and cross-linked proteins were immunoprecipitated (IP) with the cross-linkdirected antibody 81D4. Samples were then run on Western blots and probed with anti-myc antibody (which recognizes huntingtin fragments) ( $A$ ). HEK 293T cells were loaded with fura-2 $\mathrm{AM}$, and a calcium baseline was obtained. The calmodulin inhibitor w5-hydrochloride was added in increasing concentrations starting with $50 \mu \mathrm{M}(B)$. The w5-hydrochloride was not washed out before additional drug treatment and thus resulted in a final concentration of 300 $\mu \mathrm{m}$ in this experiment. The w5-hydrochloride had no effect on intracellular calcium concentrations. Each tracing represents the ratio of bound to unbound calcium in a single cell in this representative experiment. The minor depressions in the tracings at the time of drug treatment are an artifact resulting from the addition of a liquid and do not represent an actual calcium flux. There was a robust increase in intracellular calcium levels during treatment with the calcium ionophore ionomycin, which was used as a positive control (B). IB, Immunoblot.

pared with cotransfection of polyglutamine-expanded huntingtin and transglutaminase 2 (Fig. $2 A$ ).

The pathological length of huntingtin may be a trigger for transglutaminase cross-linking activity in HD. Mutant huntingtin proteins (with glutamine expansions) may bind both transglutaminase 2 and calmodulin in cells. Calmodulin-huntingtin-transglutaminase complexes may then activate transglutaminases that crosslink huntingtin fragments together, forming stable insoluble aggregates and inclusions. Calmodulin may be providing the calcium needed for activation of transglutaminase. Because huntingtin and calmodulin interact with high affinity, the calmodulin may then remain bound to huntingtin and become part of the insoluble aggregates that are formed via transglutaminase cross-linking of huntingtin. Calmodulin does not appear to be cross-linked by transglutaminase (data not shown) but is present in the insoluble 
fraction in cells transfected with htt-N63-148Q-myc and transglutaminase 2 and also is a component of intranuclear inclusions in the HD brain.

All cell-culture experiments in this study used constructs encoding $\mathrm{N}$-terminal fragments of huntingtin protein rather than full-length huntingtin protein. $\mathrm{N}$-terminal fragments of mutant huntingtin protein exist in HD cases, and although controversial, there is evidence suggesting that $\mathrm{N}$-terminal fragments rather than full-length huntingtin are present in the intranuclear inclusions in HD (DiFiglia et al., 1997; Mende-Mueller et al., 2001; Kegel et al., 2002). Transgenic mice expressing the $\mathrm{N}$-terminal fragments of mutant huntingtin protein develop pathological changes more rapidly and develop a more severe neuropathology, including more intranuclear inclusions (Li et al., 2000; Meade et al., 2002; Menalled and Chesselet, 2002). However, the relative significance of mouse models using truncated versus fulllength huntingtin constructs remains contentious. In cells in culture, truncated huntingtin protein increases aggregate formation and susceptibility to cell death (Martindale et al., 1998).

The calmodulin inhibitor w5-hydrochloride prevents crosslinking of huntingtin in cells that are cotransfected with htt-N63148Q-myc and transglutaminase 2. Intracellular calcium levels were not altered by the calmodulin inhibitor at 50-1000 $\mu \mathrm{M}$, concentrations that prevent cross-linking of huntingtin by transglutaminase 2. Therefore, the effect of the calmodulin inhibitor is specific and is not merely a result of altered calcium levels in cells.

Additionally, dysregulation of calcium homeostasis (Tang et al., 2003) may contribute to increases in calcium levels in the neuronal nucleus and cytosol, resulting in inappropriate activation of transglutaminases that leads to intranuclear inclusion formation (Cooper et al., 1999). Striatal and cortical regions may not have sufficient calcium-buffering capacity, thereby resulting in increased vulnerability of these neurons and their degeneration (Sieradzan et al., 1999). For example, levels of PEP-19, a calmodulin-binding protein, are decreased in HD and in Alzheimer's disease in selectively vulnerable brain regions (Utal et al., 1998). This finding suggests that dysregulation of calcium-calmodulin homeostasis may play a role in the pathophysiology of HD. It is possible that mutant huntingtin disrupts calmodulinmediated intracellular processes that involve PEP-19 (Utal et al., 1998). Proteins that regulate calcium homeostasis such as PEP19 , calmodulin, calbindin, and calretin bind intracellular calcium and act as intracellular calcium buffers (Baimbridge et al., 1992). These proteins may also be involved in abnormal transglutaminase activity.

Calmodulin plays a role in regulating cross-linking of proteins in the human erythrocyte cytoskeleton (Billett and Puszkin, 1991), human platelets (Puszkin and Raghuraman, 1985), and the chicken gizzard (Puszkin and Raghuraman, 1985), and may regulate transglutaminase in neurodegenerative disease. Our findings suggest that calmodulin regulates transglutaminase cross-linking of the mutant huntingtin fragments. Additional studies need to be done to confirm that calmodulin regulates the activity of transglutaminases in HD. Inhibition of calmodulin is a way of altering transglutaminase cross-linking of huntingtin in cells in culture but may not be a feasible therapeutic strategy in humans because of the host of other enzymes that calmodulin regulates, such as phosphodiesterases, adenylate cyclases, phosphatases, protein kinases, and ATPases (Hazeki et al., 2002). An important role of calmodulin is to activate nitric oxide synthases (Daff, 2003) and calmodulin kinases (Deckel, 2001). In mice transgenic for the HD mutation, there are increased levels of calmodulin kinase IV and nitric oxide synthase (Deckel, 2001) and decreased levels of calmodulin in late stages (Deckel et al., 2002). Calmodulin may be sequestered in aggregates with huntingtin protein in the HD transgenic mice, as seen in HD in this current study. Inhibiting the sequestration of calmodulin in the aggregates and the interaction of calmodulin with transglutaminase and huntingtin protein may allow calmodulin to function normally and reduce aggregate formation and stability. Calmodulin modulates calmodulin kinase IV that regulates transcription factors that may contribute to neuronal dysfunction and cell death in HD (Steffan et al., 2000; Jiang et al., 2003). Calmodulin also modulates nitric oxide synthase, which normally is involved in neurotransmission, cerebral blood flow, endocrine regulation, and other normal processes but can have a neurotoxic, oxidative stress effect under pathological conditions (Deckel et al., 2002; Montgomery et al., 2003). Overall, modulation of calmodulin may be a useful tool to determine the pathogenic mechanisms in HD.

Transglutaminase-catalyzed cross-linking of huntingtin protein fragments is a plausible mechanism of formation of insoluble aggregates of huntingtin protein and stabilization of the intranuclear inclusions found in postmortem HD tissue. Our laboratory has demonstrated previously the colocalization of huntingtin, transglutaminase 2, and the transglutaminase-catalyzed crosslink in HD cortical intranuclear inclusions (Zainelli et al., 2003). We also demonstrated that transglutaminase cross-links mutant huntingtin proteins (with polyglutamine expansions) into insoluble aggregates in cells in culture. Evidence suggests that formation of intracellular aggregates and inclusions in other neurodegenerative diseases is also attributable to transglutaminase crosslinking. For example, the neurofibrillary tangles in Alzheimer's disease (Norlund et al., 1999; Singer et al., 2002) and progressive supranuclear palsy (Zemaitaitis et al., 2000) and Lewy bodies in Parkinson's disease (Junn et al., 2003) contain transglutaminasecatalyzed cross-links. Transglutaminase-catalyzed cross-linking may also be involved in inclusion formation in other triplet repeat disorders in addition to HD (Ross et al., 1998). To prevent the seemingly detrimental activity of transglutaminase in HD and other neurodegenerative diseases, agents that inhibit transglutaminase are currently under investigation (Dedeoglu et al., 2002; Karpuj et al., 2002a,b). It has been demonstrated that the chemical inhibitor cystamine decreases transglutaminase activity, decreases aggregate formation, increases viability of cells in culture, and improves motor function in a mouse model of HD (Dedeoglu et al., 2002). In this study, we found that calmodulin is involved in regulation of transglutaminase cross-linking of the huntingtin protein, and that calmodulin may be a factor involved in intranuclear inclusion formation in HD. These studies lay the foundation for the understanding and development of novel approaches to regulate transglutaminase cross-linking and aggregate formation to increase neuroprotection in HD.

\section{References}

Baimbridge KG, Celio MR, Rogers JH (1992) Calcium-binding proteins in the nervous system. Trends Neurosci 15:303-308.

Bao J, Sharp AH, Wagster MV, Becher M, Schilling G, Ross CA, Dawson VL, Dawson TM (1996) Expansion of polyglutamine repeat in huntingtin leads to abnormal protein interactions involving calmodulin. Proc Natl Acad Sci USA 93:5037-5042.

Becher MW, Kotzuk JA, Sharp AH, Davies SW, Bates GP, Price DL, Ross CA (1998) Intranuclear neuronal inclusions in Huntington's disease and dentatorubral and pallidoluysian atrophy: correlation between the density of inclusions and IT15 CAG triplet repeat length. Neurobiol Dis 4:387-397.

Billett HH, Puszkin EG (1991) The red cell membrane contains calmodulin- 
regulated crosslinking and proteolytic activity. Hematol Pathol 5:185-193.

Cariello L, de Cristofaro T, Zanetti L, Cuomo T, Di Maio L, Campanella G, Rinaldi S, Zanetti P, Di Lauro R, Varrone S (1996) Transglutaminase activity is related to CAG repeat length in patients with Huntington's disease. Hum Gen 98:633-635.

Cheung WY (1982) Role of calmodulin in brain function. Prog Brain Res 56:237-253.

Cooper AJ, Sheu KF, Burke JR, Strittmatter WJ, Gentile V, Peluso G, Blass JP (1999) Pathogenesis of inclusion bodies in $(C A G)_{n} / Q_{n}$-expansion diseases with special reference to the role of tissue transglutaminase and to selective vulnerability. J Neurochem 72:889-899.

DaffS (2003) Calmodulin-dependent regulation of mammalian nitric oxide synthase. Biochem Soc Trans 31:502-505.

Davies SW, Turmaine M, Cozens BA, DiFiglia M, Sharp AH, Ross CA, Scherzinger E, Wanker EE, Mangiarini L, Bates GP (1997) Formation of neuronal intranuclear inclusions underlies the neurological dysfunction in mice transgenic for the HD mutation. Cell 90:537-548.

Deckel AW (2001) Nitric oxide and nitric oxide synthase in Huntington's disease. J Neurosci Res 64:99-107.

Deckel AW, Elder R, Fuhrer G (2002) Biphasic developmental changes in $\mathrm{Ca} 2+/$ calmodulin-dependent proteins in R6/2 Huntington's disease mice. NeuroReport 13:707-711.

Dedeoglu A, Kubilus JK, Jeitner TM, Matson SA, Bogdanov M, Kowall NW, Matson WR, Cooper AJ, Ratan RR, Beal MF, Hersch SM, Ferrante RJ (2002) Therapeutic effects of cystamine in a murine model of Huntington's disease. J Neurosci 22:8942-8950.

DiFiglia M, Sapp E, Chase KO, Davies SW, Bates GP, Vonsattel JP, Aronin N (1997) Aggregation of huntingtin in neuronal intranuclear inclusions and dystrophic neurites in brain. Science 277:1990-1993.

El Alaoui S, Mian S, Lawry J, Quash G, Griffin M (1992) Cell cycle kinetics, tissue transglutaminase and programmed cell death (apoptosis). FEBS Lett 311:174-178.

Gentile V, Sepe C, Calvani M, Melone MA, Cotrufo R, Cooper AJ, Blass JP, Peluso G (1998) Tissue transglutaminase-catalyzed formation of highmolecular weight aggregates in vitro is favored with long polyglutamine domains: a possible mechanism contributing to CAG-triplet diseases. Arch Biochem Biophys 352:314-321.

Gourfinkel-An I, Cancel G, Duyckaerts C, Faucheux B, Hauw J, Trottier Y, Brice A, Agid Y, Hirsch EC (1998) Neuronal distribution of intranuclear inclusions in Huntington's disease with adult onset. NeuroReport 9:1823-1826.

Gutekunst C-A, Li S-H, Yi H, Mulroy JS, Kuemmerle S, Jones R, Rye D, Ferrante RJ, Hersch SM, Li X-J (1999) Nuclear and neuropil aggregates in Huntington's disease: relationship to neuropathology. J Neurosci 19:2522-2534.

Hand D, Perry MJ, Haynes LW (1993) Cellular transglutaminases in neural development. Int J Dev Neurosci [Erratum (1994) 12:527] 11:709-720.

Hazeki N, Tung Y-C, Goto J, Kanazawa I (2000) Formic acid dissolves aggregates of an $\mathrm{N}$-terminal huntingtin fragment containing an expanded polyglutamine tract: applying to quantification of protein components of the aggregates. Biochem Biophys Res Commun 277:386-393.

Hazeki N, Tsukamoto T, Yazawa I, Koyama M, Hattori S, Someki I, Iwatsubo T, Nakamura K, Goto J, Kanazawa I (2002) Ultrastructure of nuclear aggregates formed by expressing an expanded polyglutamine. Biochem Biophys Res Commun 294:429-440.

Jiang H, Nucifora Jr FC, Ross CA, DeFranco DB (2003) Cell death triggered by polyglutamine-expanded huntingtin in a neuronal cell line is associated with degradation of CREB-binding protein. Hum Mol Genet $12: 1-12$.

Junn E, Ronchetti RD, Quezado MM, Kim SY, Mouradian MM (2003) Tissue transglutaminase-induced aggregation of alpha-synuclein: implications for Lewy body formation in Parkinson's disease and dementia with Lewy bodies. Proc Natl Acad Sci USA 100:2047-2052.

Kahlem P, Green H, Djian P (1998) Transglutaminase action imitates Huntington's disease: selective polymerization of huntingtin containing expanded polyglutamine. Mol Cell 1:595-601.

Karpuj MV, Garren H, Slunt H, Price DL, Gusella J, Becher MW, Steinman L (1999) Transglutaminase aggregates huntingtin into nonamyloidogenic polymers, and its enzymatic activity increases in Huntington's disease brain nuclei. Proc Natl Acad Sci USA 96:7388-7393.

Karpuj MV, Becher MW, Steinman L (2002a) Evidence for a role for trans- glutaminase in Huntington's disease and the potential therapeutic implications. Neurochem Int 40:31-36.

Karpuj MV, Becher MW, Springer JE, Chabas D, Youssef S, Pedotti R, Mitchell D, Steinman L (2002b) Prolonged survival and decreased abnormal movements in transgenic model of Huntington disease, with administration of the transglutaminase inhibitor cystamine. Nat Med 8:143-149.

Kegel KB, Meloni AR, Yi Y, Kim YJ, Doyle E, Cuiffo BG, Sapp E, Wang Y, Qin ZH, Chen JD, Nevins JR, Aronin N, DiFiglia M (2002) Huntingtin is present in the nucleus, interacts with the transcriptional corepressor C-terminal binding protein, and represses transcription. J Biol Chem 277:7466-7476.

Lesort M, Chun W, Johnson GV, Ferrante RJ (1999) Tissue transglutaminase is increased in Huntington's disease brain. J Neurochem 73:2018-2027.

Li H, Li SH, Johnston H, Shelbourne PF, Li XJ (2000) Amino-terminal fragments of mutant huntingtin show selective accumulation in striatal neurons and synaptic toxicity. Nat Genet 25:385-389.

Lorand L (1996) Neurodegenerative diseases and transglutaminases. Proc Natl Acad Sci USA 93:14310-14313.

Martindale D, Hackam A, Wieczorek A, Ellerby L, Wellington C, McCutcheon K, Singaraja R, Kazemi-Esfarjani P, Devon R, Kim SU, Bredesen DE, Tufaro F, Hayden MR (1998) Length of huntingtin and its polyglutamine tract influences localization and frequency of intracellular aggregates. Nat Genet 18:150-154.

Meade CA, Deng YP, Fusco FR, Del Mar N, Hersch S, Goldowitz D, Reiner A (2002) Cellular localization and development of neuronal intranuclear inclusions in striatal and cortical neurons in R6/2 transgenic mice. J Comp Neurol 449:241-269.

Menalled LB, Chesselet MF (2002) Mouse models of Huntington's disease. Trends Pharmacol Sci 23:32-39.

Mende-Mueller LM, Toneff T, Hwang SR, Chesselet MF, Hook VY (2001) Tissue-specific proteolysis of Huntingtin (htt) in human brain: evidence of enhanced levels of $\mathrm{N}$ - and C-terminal htt fragments in Huntington's disease striatum. J Neurosci 21:1830-1837.

Montgomery HJ, Bartlett R, Perdicakis B, Jervis E, Squier TC, Guillemette JG (2003) Activation of constitutive nitric oxide synthases by oxidized calmodulin mutants. Biochemistry 42:7759-7768.

Norlund MA, Lee JM, Zainelli GM, Muma NA (1999) Elevated transglutaminase-induced bonds in PHF tau in Alzheimer's disease. Brain Res 851:154-163.

Plank B, Pifl C, Hellmann G, Wyskovsky W, Hoffmann R, Suko J (1983) Correlation between calmodulin-dependent increase in the rate of calcium transport and calmodulin-dependent phosphorylation of cardiac sarcoplasmic reticulum. Characterization of calmodulin-dependent phosphorylation. Eur J Biochem 136:215-221.

Puszkin EG, Raghuraman V (1985) Catalytic properties of a calmodulinregulated transglutaminase from human platelet and chicken gizzard. J Biol Chem 260:16012-16020.

Ross CA, Margolis RL, Becher MW, Wood JD, Engelender S, Cooper JK, Sharp AH (1998) Pathogenesis of neurodegenerative diseases associated with expanded glutamine repeats: new answers, new questions. Prog Brain Res 117:397-419.

Schnell SA, Staines WA, Wessendorf MW (1999) Reduction of lipofuscinlike autofluorescence in fluorescently labeled tissue. J Histochem Cytochem 47:719-730.

Selkoe DJ, Abraham C, Ihara Y (1982) Brain transglutaminase: in vitro crosslinking of human neurofilament proteins into insoluble polymers. Proc Natl Acad Sci USA 79:6070-6074.

Sieradzan KA, Mechan AO, Jones L, Wanker EE, Nukina N, Mann DMA (1999) Huntington's disease intranuclear inclusions contain truncated, ubiquitinated huntingtin protein. Exp Neurol 156:92-99.

Singer SM, Zainelli GM, Norlund MA, Lee JM, Muma NA (2002) Transglutaminase bonds in neurofibrillary tangles and paired helical filament tau early in Alzheimer's disease. Neurochem Int 40:17-30.

Steffan JS, Kazantsev A, Spasic-Boskovic O, Greenwald M, Zhu YZ, Gohler H, Wanker EE, Bates GP, Housman DE, Thompson LM (2000) The Huntington's disease protein interacts with $\mathrm{p} 53$ and CREB-binding protein and represses transcription. Proc Natl Acad Sci USA 97:6763-6768.

Takahashi A, Camacho P, Lechleiter JD, Herman B (1999) Measurement of intracellular calcium. Physiol Rev 79:1089-1125. 
Tang TS, Tu H, Chan EY, Maximov A, Wang Z, Wellington CL, Hayden MR, Bezprozvanny I (2003) Huntingtin and huntingtin-associated protein 1 influence neuronal calcium signaling mediated by inositol- $(1,4,5)$ triphosphate receptor type 1. Neuron 39:227-239.

Trottier Y, Devys D, Imbert G, Saudou F, An I, Lutz Y, Weber C, Agid Y, Hirsch EC, Mandelkow E-M (1995) Cellular localization of the Huntington's disease protein and discrimination of the normal and mutated form. Nat Genet 10:104-110.

Utal AK, Stopka AL, Roy M, Coleman PD (1998) PEP-19 immunohistochemistry defines the basal ganglia and associated structures in the adult human brain, and is dramatically reduced in Huntington's disease. Neuroscience 86:1055-1063.
Violante V, Luongo A, Pepe I, Annunziata S, Gentile V (2001) Transglutaminasedependent formation of protein aggregates as possible biochemical mechanism for polyglutamine diseases. Brain Res Bull 56:169-172.

Vonsattel JP, Myers RH, Stevens TJ, Ferrante RJ, Bird ED, Richardson EP (1983) Neuropathological classification of Huntington's disease. J Neuropathol Exp Neurol 44:559-577.

Zainelli GM, Ross CA, Troncoso JC, Muma NA (2003) Transglutaminase cross-links in intranuclear inclusions in Huntington disease. J Neuropathol Exp Neurol 62:14-24.

Zemaitaitis MO, Lee JM, Troncoso JC, Muma NA (2000) Transglutaminaseinduced cross-linking of tau proteins in progressive supranuclear palsy. J Neuropathol Exp Neurol 59:983-989. 